\title{
Irreversible Dynamics: A topical issue dedicated to Paul Manneville
}

\author{
Published online: 21 April 2014 - (c) EDP Sciences / Società Italiana di Fisica / Springer-Verlag 2014
}

The study of classical physics underwent a revolution in the 1970s-1980s. A watershed event was the discovery of deterministic chaos in a model of thermal convection by Edward Lorenz in 1963 [1], followed by the introduction of strange attractors and transition scenarios by David Ruelle and Floris Takens in 1971 [2]. The concurrent revival and extension of the theory of dynamical systems encompasses spatiotemporal chaos and coupled oscillator arrays. Applications to fluid dynamics range from pattern formation in convection to the onset of turbulence in shear flows. Originally focused on fluid and solid mechanics, this approach cuts across the usual boundaries of scientific domains and has proved equally useful in understanding reaction kinetics, biology, and condensed matter physics.

Fifty years after the ground-breaking paper of Lorenz, this is the theme of the mini-colloquium Irreversible Dynamics which focused on the contributions of Paul Manneville and his collaborators, and was held in Paris as part of the Rencontres du Non Linéaire in March 2013. This colloquium was the occasion to solicit original articles which are now assembled in this Topical Issue of the European Physical Journal E dedicated to Irreversible Dynamics, with a wide interpretation of this field in the spirit that Paul Manneville has always defended.

As an engineering student at the École Centrale of Paris at the end of the 1960s, Paul Manneville attended lectures of Raymond Jancel on dynamical systems at the Institut Henri Poincaré. Discovering Vladimir Arnold's work, Paul boldly chose ergodic theory as the subject of his engineering thesis in 1969. He next gained a diplôme d'études avancées (advanced studies diploma) in solid state physics at the Laboratoire de Physique des Solides (LPS-Orsay) of the University Paris Sud directed by Jacques Friedel, and continued his training in this field with an experimental study of low-temperature magnetic order at the Service de Physique du Solide et de Résonance Magnétique of the Commissariat à l'Énergie Atomique (CEA) at L'Orme des Merisiers in a group led by Jacques-Michel Hammann. It was there, in 1972, that he completed his thèse de doctorat de $3^{e}$ cycle from the University Pierre et Marie Curie. Subsequently, he spent his year of military service in a scientific position at LPS-Orsay under the supervision of Jean Charvolin, studying molecular mobility in liquid crystals by magnetic resonance. A phase transition took place in 1973, when he became the father of twins, Jean-Baptiste and Sébastien: the incompatibility of combining low-temperature experiments in his former group with caring for small children led Paul towards more theoretical research and so he changed his affiliation at L'Orme for a group led by Nino Boccara and Gobalakichena Sarma. It is fortunate that the young father was able to transmit his passion for the physical sciences to his twin sons, who participated in the Rencontres du Non Linéaire by relating their most recent discoveries, on the effects of mechanical stresses in cellular transport for Jean-Baptiste, and on the instabilities of complex fluids for Sébastien, whose article on the shearing of solutions of wormlike micelles is published in this Topical issue.

At L'Orme, Paul developed a model of superconducting wires coupled by the Josephson effect displaying a divergence of critical fields, and this was the topic of his first solo paper. But the wild imaginings of theorists may crash into the hard physical reality of a given time, and indeed, Pierre-Gilles de Gennes warned him of the difficulty of synthesizing such materials. The predictions were, however, partly verified years later and the model could also be adapted to describe the stacking of discotic liquid crystals. Again advised by de Gennes, Paul collaborated with Étienne Guyon, Élisabeth Dubois-Violette and Pawel Pieranski at the neighbouring LPS-Orsay to study the stability of flows in liquid crystals. Still fascinated by liquid crystals, Pieranski has shown us in this issue his latest investigations, following in the footsteps of ... a spider! Paul's interest in non-equilibrium phenomena motivated him to develop a

\footnotetext{
* Contribution to the Topical Issue "Irreversible Dynamics: A topical issue dedicated to Paul Manneville", edited by Patrice Le Gal and Laurette S. Tuckerman.
} 
detailed theory of linear shear instabilities in nematics and he began to address their nonlinear saturation. Bifurcation theory and perturbation expansions about equilibria observed in the LPS experiments became part of his thèse de doctorat d'État, which he defended in 1977 after being selected as an attaché de recherche at the Centre National de la Recherche Scientifique (CNRS) in 1976. At this time, the group of Pierre Bergé had decided to turn to the physics of hydrodynamic structures and transition to turbulence. Growing in influence, the group at L'Orme attracted a number of physicists such as Christiane Normand, Manuel Velarde and Eduardo Wesfreid. Wesfreid, along with researchers from his group at PMMH, has contributed to this issue a detailed analysis of transitional flows in Poiseuille flow.

At the end of the 1970s, inviting Paul to give a tutorial course on instabilities in liquid crystals at Les Houches, Yves Pomeau encouraged him to turn to the new theories of transition to chaos and their applications to hydrodynamics. The collaboration with Pomeau led to the publication of a pair of very influential papers in Physics Letters in 1979 [3] and Communications in Mathematical Physics in 1980 [4]. Based on the first observations of "bursts" in numerical simulations of the Lorenz attractor, these articles defined the three types of intermittency and the associated transition scenarios towards chaos. At this time, they have been cited over 1000 times! In this topical issue, Pomeau and his collaborators present a study of the singularities of the almost instantaneous (at the timescale of the universe) collapse of supernovae using the tools of bifurcation theory. The scenario of transition to chaos via intermittency was afterwards observed in the experiments of Bergé and Monique Dubois, confirming the viewpoint of the CEA group leader promoting a symbiotic relationship between theorists and experimentalists.

In the beginning of the $1980 \mathrm{~s}$, research on convection turned to extended systems in which the structures are relatively unconfined by boundaries. Paul continued his collaboration with Pomeau together with Stéphane Zaleski, Alain Pumir and Jean-Michel Piquemal, developing theories of hydrodynamic structures: amplitude equations, phase diffusion, and analysis of defects. Experimentalists were not to be outdone as Vincent Croquette led the experimental group towards extended systems. During our mini-colloquium dedicated to Paul, Pumir and Croquette presented their most recent results, both on biological systems. While the CEA group was further reinforced by the arrival of Alain Pocheau and then of L.T. and of P.L.G. in 1983, Paul played the role of a leading sage, achieving remarkable advances by clever projections of the Boussinesq equations. Retaining an interest in chaotic systems, in particular during the thesis work of Philippe Ghendrih and the investigations by Croquette of his famous chaotic compass, Paul developed analytical techniques which could describe the nonlinear dynamics of grain boundaries, sliding of dislocations, or wavelength selection, all of which were observed experimentally in convective patterns in extended geometries. Ghendrih and his collaborators at Cadarache present in this issue of EPJE the latest results of their modelling via avalanches of bursts in plasma turbulence, while Pocheau and Benoit Roman present the astonishing behaviour of the mechanics of buckling in thin plates.

In the 1980s as well, Paul polished the notes of the courses he gave at the Physics of Liquids Masters program of University Pierre et Marie Curie and at the Summer School in Condensed Matter Physics at Beg Rohu (Quiberon, France), which he championed along with its founder, Claude Godrèche. These notes would become the book Dissipatives Structures and Weak Turbulence, published by Academic Press in 1990, a monograph that continues to inspire and serves as an important reference for classroom presentations. He later wrote another book, drawn from the class on nonlinear dynamics he taught at the École Nationale Supérieure des Techniques Avancées (ENSTA), Instability, chaos and turbulence (éditions de l'École Polytechnique, distribution Ellipses, 2004 and 2010). The arrival of Hugues Chaté as a thesis student led to the study of systems with a very large number of degrees of freedom. Model differential equations, networks of coupled maps, and cellular automata became the playground of Paul and Chaté, who used numerical simulations to study the appearance of collective behaviour and of transitions to spatiotemporal chaos. They co-directed the thesis of Philippe Marcq, who has contributed an article on a model of active complex fluids reproducing certain properties of cellular layers, and then that of Anaël Lemaître, whose oral presentation was on avalanches of plastic deformations in amorphous constrained materials. At the colloquium, Chaté presented a review of his work with Paul on collective behaviour of systems with a very large number of degrees of freedom.

In 1994, Paul joined LadHyX, a laboratory newly founded by Patrick Huerre, who presented recent analyses of instabilities of jets and plumes at this colloquium. During this period, Paul also investigated the instabilities of falling liquid layers with Christian Ruyer-Quil who contributed to this volume an analysis of the bifurcations present in these flows. Paul's priorities turned to the experiments carried out at L'Orme by François Daviaud, Olivier Dauchot and their students, Sabine Bottin and Arnaud Prigent, on cylindrical and plane Couette flow. Indeed, although the subcritical transition to turbulence undergone by these sheared flows has been studied for over a century since Reynolds' first investigations, fundamental questions still remain. Paul has dedicated considerable energy to this problem and has played a central role in a community of researchers centred on the plateau of Saclay. Continuing his research on turbulent systems, Daviaud presented at the colloquium the latest results obtained on transitions in turbulent swirling Kármán flow, while Dauchot gave a new interpretation of subcritical transitions in shear flows in terms of a glass transition. Applying the methods he developed while studying convective structures in extended geometries, Paul then supervised the theses of Maher Lagha on the modelling of turbulent spots in plane Couette flow and of Joran Rolland on the analysis via numerical simulations of statistical properties of alternating turbulent and laminar bands. 


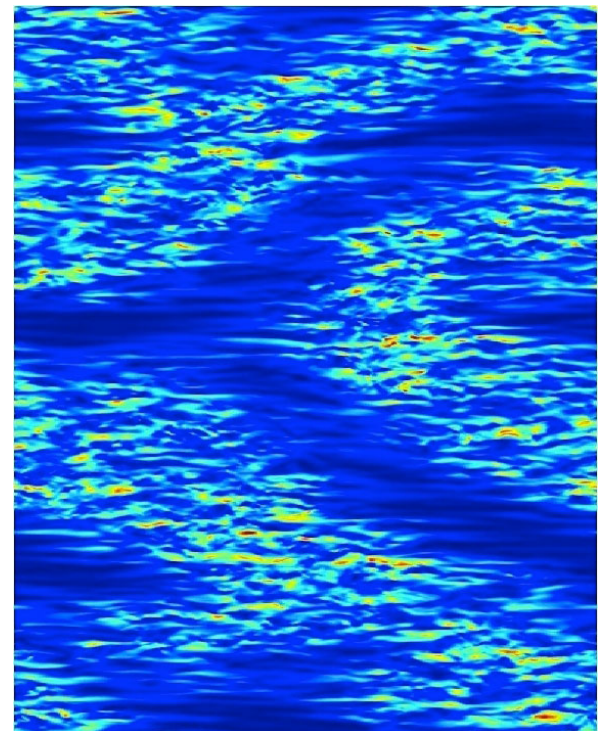

Fig. 1. Image of a dislocation in a laminar-turbulent pattern obtained by numerical simulation of plane Couette flow, the flow between two parallel counter-translating plates. The local intensity of turbulence is displayed in color levels, blue corresponding to laminar flow and red to highly distorted flow. The streamwise (spanwise) direction is horizontal (vertical). Such patterns appear between the global stability threshold below which the flow unconditionally returns to laminar and an upper threshold above which turbulence has essentially uniform intensity. The wavelength of the pattern, the origin of which is still mysterious, is very large when compared to the distance between the plates revealed by the smallest scales in the spanwise direction. Simulation by Paul Manneville using Gibson's ChannelFlow code (http://channelflow.org/). For technical details, see [5].

He suggested that a Turing instability might explain these experimentally observed banded patterns that he had reproduced numerically; see fig. 1 .

A scientific descendent of de Gennes and Bergé, Paul has retained throughout his career a keen interest in research combining experiments and advanced theoretical analysis. He has authored many articles that are genuine breakthroughs on topics ranging from the theory of deterministic chaos to the collective behaviour of spatially extended systems, and the transition to turbulence in convection cells or shear flows. As can be seen in this topical issue, many of his students and collaborators today carry out research whose great diversity demonstrates the richness of nonlinear physics and irreversible dynamics. Paul's passion, rigor, and collegiality are examples for all who have spent time in his scientific orbit, represented by this topical issue which is completed by the articles of our colleagues Yohann Duguet, Bruno Eckhardt, Dan Henningson, Uwe Thiele, Alessandro Bottaro and Innocent Mutabazi and their colleagues who have joined us in this friendly tribute to Paul Manneville.

\section{References}

1. E.N. Lorenz, J. Atmos. Sci. 20, 130 (1963).

2. D. Ruelle, F. Takens, Commun. Math. Phys. 20, 167 (1971).

3. P. Manneville, Y. Pomeau, Phys. Lett. 75, 1 (1979).

4. Y. Pomeau, P. Manneville, Commun. Math. Phys. 74, 189 (1980).

5. P. Manneville, Fluid Dyn. Res. 43, 065501 (2011).

Patrice Le Gal and Laurette S. Tuckerman 